\title{
Identification of amino acids related to catalytic function of Sulfolobus solfataricus P1 carboxylesterase by site-directed mutagenesis and molecular modeling
}

\author{
Yun-Ho Choi ${ }^{1, \# \#}, Y e-N a$ Lee ${ }^{1, \#}$, Young-Jun Park ${ }^{2}$, Sung-Jin Yoon ${ }^{2} \mathcal{E}$ Hee-Bong Lee ${ }^{1, *}$ \\ ${ }^{1}$ Department of Biochemistry, College of Natural Sciences, Kangwon National University, Chuncheon $24341,{ }^{2}$ Metabolic Regulation \\ Research Center, Korea Research Institute of Bioscience and Biotechnology, Daejeon 34141, Korea
}

\begin{abstract}
The archaeon Sulfolobus solfataricus P1 carboxylesterase is a thermostable enzyme with a molecular mass of $33.5 \mathrm{kDa}$ belonging to the mammalian hormone-sensitive lipase (HSL) family. In our previous study, we purified the enzyme and suggested the expected amino acids related to its catalysis by chemical modification and a sequence homology search. For further validating these amino acids in this study, we modified them using site-directed mutagenesis and examined the activity of the mutant enzymes using spectrophotometric analysis and then estimated by homology modeling and fluorescence analysis. As a result, it was identified that Ser151, Asp244, and His274 consist of a catalytic triad, and Gly80, Gly81, and Ala152 compose an oxyanion hole of the enzyme. In addition, it was also determined that the cysteine residues are located near the active site or at the positions inducing any conformational changes of the enzyme by their replacement with serine residues. [BMB Reports 2016; 49(6): 349-354]
\end{abstract}

\section{INTRODUCTION}

Esterases (EC 3.1.1.x) are hydrolases that catalyze the cleavage of the ester bond, and they are widely distributed in all living organisms, Bacteria, Archaea, and Eukarya (1). These ester hydrolases include two major groups: carboxylesterases (EC 3.1.1.1), which hydrolyze carboxyl esters with short chains $(<$ 10 carbon atoms), and lipases (EC 3.1.1.3), which hydrolyze acylglycerols with long chains ( $\geq 10$ carbon atoms) (2).

Bacterial esterases are classified into 14 families (I to XIV) mainly based on the comparison of their amino acid sequences

${ }^{*}$ Corresponding author. Tel: +82-33-250-8512; Fax: +82-33-2595664; E-mail: leehbong@kangwon.ac.kr

${ }^{\text {"}}$ These authors contributed equally to this work.

http://dx.doi.org/10.5483/BMBRep.2016.49.6.077

Received 7 May 2016, Revised 17 May 2016, Accepted 17 May 2016

Keywords: Carboxylesterase, Molecular modeling, Site-directed mutagenesis, Sulfolobus solfataricus P1, Thermostability and some fundamental biological properties $(3,4)$. Among these families, archaeal esterases are known to be grouped into only two families, IV and V. Most archaeal esterases belong to family IV, which show high amino acid similarity to the mammalian hormone-sensitive lipase (HSL) family (5). Esterases belonging to the $\alpha / \beta$ hydrolase superfamily are known to contain a typical catalytic triad consisting of Ser, Asp, and His residues and a conserved pentapeptide, Gly-x-Ser-x-Gly (where $\mathrm{x}$ is any amino acid residue), containing the serine residue of the catalytic triad $(2,6,7)$. Recently, the number of structural studies of esterases has increased remarkably with the analysis of their gene sequences and crystal structures together with active studies of their genomes. However, as compared to the large numbers of crystal structures of bacterial esterases, only a few crystal structures of esterases from archaea such as Archaeoglobus fulgidus (8), hot springs in Indonesia (9), Pyrobaculum calidifontis VA1 (10), and Sulfolobus tokodaii (11) were known until now.

Esterases have attracted enormous attention because of their industrial applications in detergents, food, dairy, diagnostics, and biotransformation (12). Most esterases used in industry are bacterial enzymes. However, their use in industrial processes has many restrictions at high temperatures or in the presence of various chemicals like organic solvents. Hence, esterases from hyperthermophilic archaea dwelling in extremely harsh conditions are good candidates in industrial applications.

Sulfolobus solfataricus P1 (DSM1616) used in this study is a hyperthermophile belonging to the thermoacidophilic archaeon, that inhabits sulfur rich volcanic hot springs in a hightemperature $\left(>75^{\circ} \mathrm{C}\right)$ and low-pH $(<\mathrm{pH} 4)$ environment (13). We reported in previous paper that a new carboxylesterase from this archaeon S. solfataricus P1 (SsoP1Est) was purified and several biochemical properties of the enzyme were characterized, and then, its gene was identified and expressed stably in E. coli (14). Particularly, in this paper, we suggested the following from the examination of the amino acids presumed to be involved in the catalytic mechanism of the enzyme by a chemical modification study and sequence alignment with homologous esterases. First, Ser, His, and Cys residues may be related to the catalytic activity of the enzyme, as modifications of the enzyme with Ser-specific inhibitors [phenylmethylsulfonyl

ISSN: 1976-670X (electronic edition)

Copyright (C) 2016 by the The Korean Society for Biochemistry and Molecular Biology

(c) This is an open-access article distributed under the terms of the Creative Commons Attribution Non-Commercial License (http://creativecommons.org/licenses/by-nc/4.0) which permits unrestricted non-commercial use, distribution, and reproduction in any medium, provided the original work is properly cited. 
fluoride (PMSF) and diisopropylfluorophosphate (DFP)], a His-specific inhibitor [diethyl pyrocarbonate (DPC)], and Cys-specific inhibitors $\left[\mathrm{HgCl}_{2}\right.$ and $p$-chloromercuribenzoate (PCMB)], inactivated the enzyme. Second, the SsoP1Est belongs to the HSL family exhibiting a classic $\alpha / \beta$ hydrolase fold by

Table 1. Specific activities of mutant carboxylesterases by each substitution of amino acid residues

\begin{tabular}{lcc}
\hline Carboxylesterase & Sp. act. (U/mg) & Relative activity (\%) \\
\hline Wild type & 167.00 & 100 \\
Ser151Ala & 0.07 & 0.04 \\
Asp244Asn & 2.59 & 1.55 \\
His274Asn & 0.90 & 0.54 \\
Gly80Glu & 23.54 & 14.09 \\
Gly81Glu & 28.28 & 16.94 \\
Ala152Phe & 21.42 & 12.83 \\
Cys94Ser & 19.87 & 11.90 \\
Cys101Ser & 52.68 & 31.55 \\
Cys103Ser & 29.98 & 17.95 \\
Cys184Ser & 28.28 & 16.93 \\
\hline
\end{tabular}

sequence alignment with homologous esterases, which were well characterized in the structure. Thus, it contains a typical catalytic triad of esterases, which is expected to be composed of Ser151, Asp244, and His274, and another typical oxyanion hole, which is presumed to be composed of Gly80, Gly81, and Ala152.

Hence, to clarify the roles of these amino acids expected to be related to the catalytic process of the SsoP1Est reported in the previous paper, we constructed the mutant enzymes by site-directed mutagenesis, examined the catalytic activity of these mutant enzymes, and suggested their roles using the homology modeling method and fluorescence analysis.

\section{RESULTS AND DISCUSSION}

\section{Identification of mutant plasmids and purification of native and mutant SsoP1Ests}

The correct mutations of the mutant plasmids containing the SsoP1Est gene mutated at the desired positions, as shown in Table S1 in the Supplemental data, were identified by DNA sequencing (data not shown).

The native SsoP1Est used as the control was expressed in $E$. coli, and the expressed enzyme was purified 19.35-fold with a

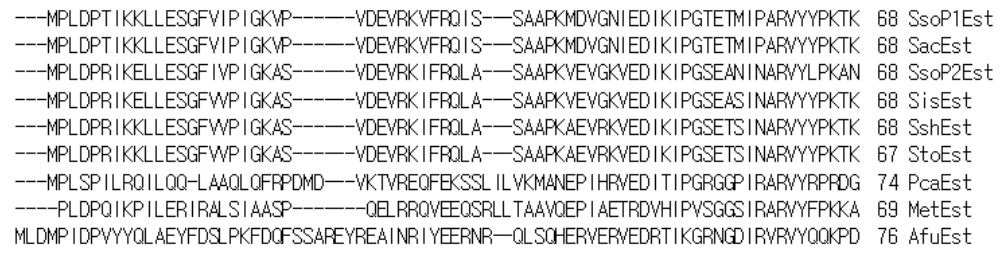

$$
\downarrow
$$

GPYGILVYLHGGFVIGDIESYCPVCRAITNAOVWASIDYRLAPENFPSAWDSVATKWIYENAESLEGKOG-VAV 147 SSOP1ESt GPYGILWYLHGGGFYIGD IESYDPYCRA I TNACNCWASIDYRLAPENKFPSAWVSYDATKNIYENAESLEGKOG-VAV 147 SaCEst GPYGVL IYLHGGGFVIGDVESYDPLCRAI INAACNCWWSVDYRLAPEYKFPSAVIDSFDATNWWYNNLDKFDGKMG-VAI 147 SsoP2Est GPYGILWYHGGGFVIGD IESYDPYCRAI INACNCWASIDYRLAPENKFPSAVIDSFDATNWWYNNLDKFDGKMG-VAI 147 SisEst GPYGILWYHGGGFYIGD IESYDPYCRA I TNACNCWASI DYRLAPENKFPSAVI DSFDATNWIYNNLDKFDCEMG-IAI 147 SshEst GPYGVLYYHGGGFYLGDIESYDPLCRA I TNSCOCVTISVDYRLAPENKFPAAWDSFDALKWNYNNSEKFNGKYG-IAV 147 StoEst ERLPAWYYHGGGFVLGSYETHDHYCRRLANLSGAWWSVDYRLAPEHKFPAAVEDAYDAAKWWVADNYOKLGVDNGK IAV 154 PcaEst AGLPAVLYYHGGGFYFGS IETHDHICRRLSRLSDSWWVDYRLAPEYKFPTAVEDAYAALKWVADRADELGVPDRIAV 149 MetEst S--PVLWYHGGGFYICSIESHDALCRRI ARLSNSTWWVVYRLAPEHKFPAAWYOCYDATKWVAENAEELRIDPSKIFV 154 AfuEst

GGDSAGGNLSAWSILTKGK--INLKHOM-IYPATG-ADCTSRSM--YEYY--DGYFL TREOIEWFGSOYLRSYMLLD 219 SsoP1Est GGDSAGGNLSAWSILTKGK--INLKHOVL-IYPATG-ADYTSRSM-VEY--DGYFL TREOIEWFGSOYLRSYMDLLD 219 SacEst AGDSAGGNLAAWALLSKGK--LNLKYOIL-IYPAVG-FDSVSRSM--IEYS--DGFFL TREIIEWFGSOYLRSPADLLD 219 SsoP2Est AGDSAGGNLAAWALLSKGK--LNLKYQIL-IYPAVG-FDNVRSM--IEYS--DGFFL TREIIEWFGSOYLRSPADLLD 219 SisEst AGDSAGGNLAAWALLSKGK--LDLKYOIL-IYPAVG-FDSYSRSM--IEYS--DGFFLTREHIEWFGSOYLRSPADLLD 219 SshEst GGDSAGGNLAAVTAILSKKEN-IKLKYOVL-IYPAVS-FDLITKSL--YDNG--EGFFL TREUIDWFGOYYRSSFADLLD 221 StoEst AGDSAGGNLAAVTAI IMARDRGESFYKYOVL-IYPAVNLTGSPTVSR--VEYSGPEYVILTADLMAMFGROYFSKPODALS 231 PcaEst AGDSAGGNLAAWSILDRNSGEKL WKOVL-IYPWNXTGYPTASL--VEFGVAETTSLPIE XVWFGROYLKRPEEAYD 226 MetEst GGDSAGGNLAAAVSIMARDSGEDFLENLPPALI I TAEYDPLRDEGE---VFGOMLRRAGVEASIVRYRGVLHGF INYYPV 231 AfuESt

IKFSP ILNODL TGLPPAL IITAEYDPL RDOGEAYANKLL MAG IPVTSVRFNVIHGFVSFFPL IPOGMDA IGL I GATLRRVFYNSF 305 SSOP1Est

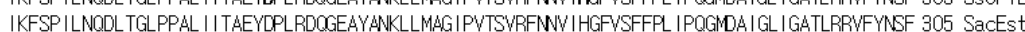
FRFSP ILVODLSGLPPAL I I TAEYCPLRDOGEAYANRLLOAGYPVVTSVRFNVI HGFLSFFPL I EOGRDA IGL I GSVLRRAFYCKS 305 SsoP2Est FRFSP I VVOLLSGLPPAL I I TAEYCPLRDOGEAYANRLLOAGVPVISVRFN W I HGFLSFFPL I EOGRDA IGL I GSVLRRAFYDKS 305 SisEst FRFSP I AODLSGLPPAL I I TAEYCPLRDOGEAYANRLLOAGYPVTSYRFNVI HGFL SFFPL IDOGKDAIGLIGSVLRRTFYDKS 305 SshEs FRFSP IL-ADLNDLPPAL II-AEHLPLRDOGEAYANKLLOSGVOVTSVRFNNVI HGFVSFFPF I EOGROA IGLIGYVLRKVFYGK- 303 StoEst

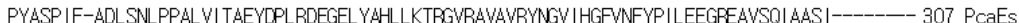
FKASPLL-ADLGGLPPALWTAEYCPLRDEGEL YAYKXKASGSRAVVAVRFAGXYHGFVSFYPFVDAGREALDLAAASIRSGLOPS- 311 MetEst LKAARDA IKHOIL I YPWWFVAPTPSLLEFGEGLWILDOK IMSWFSEOYFSREED--KFNPLASVIFAD INOIAALLVFD--- 311 AfuEst
Fig. 1. Amino acid sequence alignment of HSL-like carboxylesterase (Est) from S. solfataricus P1 (SsoP1), S. solfataricus P2 (SsoP2), S. islandicus (Sis), S. shibatae (Ssh), S. tokodaii (Sto), P. calidifontis (Pca), metagenomic library (Met), and A. fulgidus (Afu). Amino acid residues that consist of a catalytic triad and an oxyanion hole are indicated by diamonds and stars, respectively. The cysteine residues are indicated by arrows. 
yield of $40 \%$ to apparent homogeneity from cell extracts. The apparent homogeneity of the purified native and mutant enzymes were confirmed on SDS-polyacrylamide gel, and the molecular mass of the purified native and mutant enzymes was all the same, approximately $34 \mathrm{kDa}$, as that of the recombinant SsoP1Est as described previously (14) (data not shown).

\section{Catalytic activity of mutant SsoP1Ests}

In previous studies, the residues Ser151, Asp244, and His274 were tentatively identified as the catalytic triad of the native SsoP1Est by chemical modification experiments using chemical inhibitors for specific amino acids and sequence alignment with homologous esterases $(7-11,14)$. Based on these findings, in the present investigation we have produced the mutants Ser151Ala, Asp244Asn, and His274Asn. The activity of the purified mutant carboxylesterases was measured. The native carboxylesterase, SsoP1Est, was used as a control. As expected, the mutation of these three amino acids almost completely inactivated the enzymes (Table 1). These results indicate that each of the three amino acids is crucial to the enzymatic catalysis and that the catalytic triad in the SsoP1Est consists of the residues Ser151, Asp244, and His274. These results that Ser, Asp, and His are crucial to the catalysis of the enzyme were the same as those from other esterases in our previous studies using site-directed mutagenesis $(15,16)$.

In addition, to confirm whether Gly80, Gly81, and Ala152, as expected from sequence alignment with other homologous esterases showing high sequence identities (Fig. 1) and as well characterized in previous studies (14), are the amino acids that make up the oxyanion hole stabilizing a short-lived oxyanion by hydrogen bonding, these three amino acids were mutated by site-directed mutagenesis to Gly80Glu, Gly81Glu, and Ala152Phe, respectively. The result obtained from the activity measurements of these mutant carboxylesterases is shown in Table 1. Compared to the control, the activities of the mutant enzymes showed less than $17 \%$. These results indicate that all three amino acids make up the oxyanion hole and are necessary for the catalytic process of the enzyme. Hence, these amino acids are also conserved well in other esterases $(7-11,14)$, as shown in Fig. 1.

\section{Identification of roles of cysteines in SsoP1Est}

To examine the roles of cysteine residues in SsoP1Est because the modification of cysteines by cysteine-specific reagents, $\mathrm{HgCl}_{2}$ and $\mathrm{PCMB}$, significantly inactivated the enzyme in a previous study (14), each cysteine residue (Cys94, Cys101, Cys103, and Cys184) in the enzyme was mutated by site- directed mutagenesis to Cys94Ser, Cys101Ser, Cys103Ser, and Cys184Ser, respectively. As shown in Table 1, each mutation of these four cysteines in the enzyme led to remarkable inactivation of the enzyme, although the Cys101Ser mutant enzyme showed a little less inhibition $(68 \%)$ than the other mutants $(>82 \%)$ as compared to the control. The result that the substitution of each cysteine residue with serine residue greatly decreases the catalytic activity of the enzyme indicates that these cysteine residues may be present at and/or near the active site of the enzyme or that the substitution of cysteines with serines might induce the conformational change in the active site of the enzyme due to the difference in polarity between cysteine and serine.

\section{Amino acid sequence comparison}

To elucidate the above inhibitory results of the replacement of cysteines by serines, we compared the amino acid sequence of the SsoP1Est with those of other known archaeal esterases in the database. As shown in Fig. 1, the amino acid sequence of the SsoP1Est showed high identities with those of other known

\section{A}
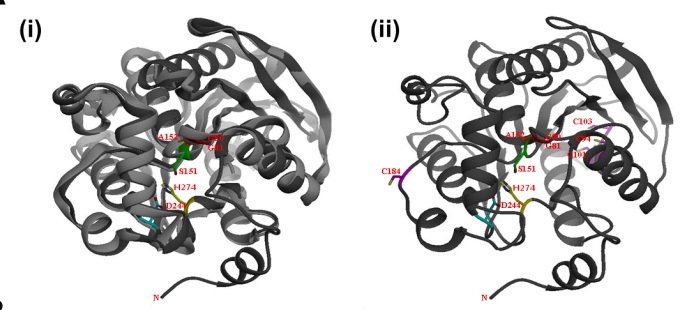

(i)

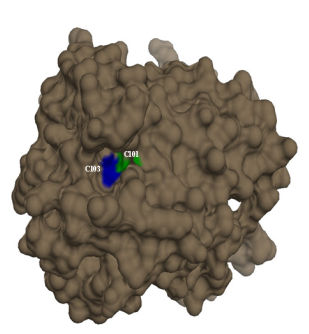

(ii)

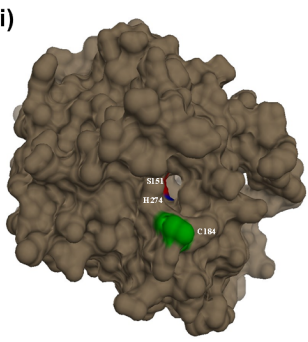

C

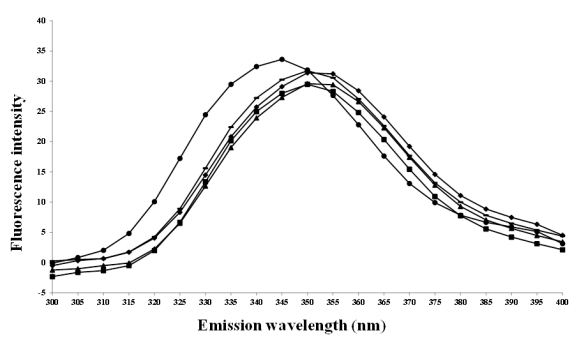

Fig. 2. (A) Homology model of SsoP1Est. Amino acids consisting of the catalytic triad and oxyanion hole are exhibited. (i) The ribbon structure of SsoP1Est (light gray) superimposed onto StoEst (gray) used as a template, and (ii) the ribbon structure of SsoP1Est including four cysteine residues. (B) The cysteine residues on the surface of SsoP1Est using "hydrophobicity surface" program. (i) Cys101 (green) and Cys103 (blue), (ii) Cys184 (green) with Ser151 (red) and His 274 (blue) consisting of a catalytic triad appeared inside the substrate entrance/product exit tunnel. (C) Emission spectrum from steady-state tryptophan fluorescence of normal SsoP1Est and mutant esterases. Normal esterase (0), mutant esterases: Cys94Ser $(\boldsymbol{\square})$, Cys101Ser $(\boldsymbol{\Delta})$, Cys103Ser $(\boldsymbol{\square})$, and Cys194Ser $(\nabla)$. Protein concentrations were $0.1 \mathrm{mg} / \mathrm{ml}$. 
esterases from the same Sulfolobus genus: The esterase from $S$. acidocaldarius (SacEst) with 99\% identity was the most similar, and the next were those from S. solfataricus P2 (SsoP2Est, 77\%), S. shibatae (SshEst, 77\%), S. islandicus (SisEst, 77\%), S. solfataricus P1 (DSM 5354; 75\%), and S. tokodaii (StoEst, 70\%), but it showed low identities with those of other archaeal esterases from $P$. calidifontis (PcaEst, 46\%), the metagenomic library (MetEst, 43\%), and A. fulgidus (AfuEst, 34\%). In particular, three cysteines (Cys94, Cys101, and Cys103) of the four cysteines in the SsoP1Est were conserved in esterases from this same Sulfolobus genus. On the contrary, only one cysteine (Cys94) was conserved in all other archaeal esterases (Fig. 1) and in all esterases belonging to the HSL-family. This result that more cysteines were conserved in esterases from the Sulfolobus seems to be related to the surrounding condition of the Sulfolobus genus dwelling in sulfur-rich hot springs. In addition, the substitution of the Cys94 by serine residue could be assumed to significantly affect the catalytic activity of the enzyme due to the $88.1 \%$ inhibition as compared to the native enzyme (Table 1 ). Although it is difficult to explain the exact reason for this inhibition, the higher polarity of the serine residue than the cysteine residue seems to induce the conformational change of the active site in the enzyme because of the easy formation of a hydrogen bond with surrounding residues (Fig. 2B). The inhibition by the mutation of Cys101Ser or Cys103Ser can also be explained by the same reason and by the disruption of the disulfide bond between them, as described previously. However, it remains in question, because these two cysteine residues may not be critical for the catalysis of the enzyme, seeing that AfuEst and MetEst have all serine residues instead of these two cysteine residues in all other esterases from the Sulfolobus genus, including SsoP1Est (Fig. 1).

\section{Homology model of SsoP1Est}

The confidence score of the three-dimensional (3D) models generated by the I-TASSER Server from multiple threading alignments and iterative assembly simulations was 1.56, meaning high reliability among the values from -5 to 2 . As a result, the I-TASSER Server finally presented ten crystal structures on the basis of the Protein Data Bank (PDB) databases reported previously: AfuEst (PDB code: 1jji), Bacillus subtilis Brefeldin A esterase (1jkm), Rhodococcus sp. heroin esterase (1|zl), Alicyclobacillus acidocaldarius esterase (1qz3), MetEst (2c7b), StoEst (3aik), Mycobacterium marinum esterase (3qh4), PcaEst (3zwq), bacterial HSL from a metagenomic library (4j7a), and $E$. coli acyl esterase ( $4 \mathrm{krx}$ ). In addition, it indicated as a result that the predicted function of the enzyme is a carboxylesterase (EC 3.1.1.1).

Four structures of these ten crystal structures are those of archaeal esterases-1jji (8), 2c7b (9), 3aik (11), and 3zwq (10). Among these four crystal structures, the StoEst (3aik) was selected and used as a template of homology modeling for SsoP1Est. This was because the StoEst is classified into the same Sulfolobus genus and the same HSL family, and it shows the highest sequence identity (68\%) with the SsoP1Est (Fig. 1). Based on the best template, StoEst (3aik), the expected structural model of SsoP1Est was created by homology modeling as the superimposed ribbon structure onto it using the UCSF Chimera 1.9 program, as shown in Fig. 2A(i). The locations of Ser151, Asp244, and His274 consisting of the catalytic triad and Gly80, Gly81, and Ala152 forming the oxyanion hole in the SsoP1Est were identified (Fig. 2A(i) and (ii)). In addition, the positions of the four cysteine residues (Cys94, Cys101, Cys103 and Cys184) in SsoP1Est were also exhibited (Fig. 2A(ii)). Particularly, the Cys184 existing only in the SsoP1Est seemed to be located at the surface/outside of the enzyme far away from the other three cysteine residues.

To determine why each mutation of Cys94Ser, Cys101Ser, Cy103Ser, and Cys184Ser inactivated the enzyme, their locations were examined with the colored substitutes of these cysteine residues using the "hydrophobicity surface" program in the UCSF Chimera 1.9 program. As shown in Fig. 2B(i) and (ii), Cys101, Cys103, and Cys184 were observed at the surface of the enzyme, but Cys94 was not. Therefore, Cys94 is located inside the enzyme and seems to be located at or near the active site of the enzyme of which the conformational change can be induced, because the Cys94Ser substitution inactivated the enzyme. Otherwise, this inactivation might be deduced by the enhanced polarity of Ser causing a conformational change around the Cys residue that propagates through the backbone to the active site. On the other hand, Cys101 and Cys103 are located at the surface of the enzyme (Fig. 2B(i)), and the inactivation by the Cys101Ser or Cys103Ser substitution seems to be due to the conformational change inside the active site of the enzyme by the enhanced polarity of Ser or by the disruption of the possible disulfide bridge formed between them, although there is no specific reported evidence for the disulfide bridge between them. Conversely, Cys184 is also observed on the surface of the enzyme (Fig. 2B(ii)), but the reason the Cys184Ser replacement inactivated the enzyme seems to be a little different from the other cysteines. This is because the Cys184 is located at the entrance/exit of the tunnel for the contact of the substrate to the active site or for the release of the products from the active site and because the amino acids, Ser151 and His274, composing the catalytic triad of the enzyme can be seen very well through the hole together with the Cys184 located at the entrance/exit.

Hence, the modification of Cys184 by Cys-specific inhibitors, $\mathrm{HgCl}_{2}$ and $\mathrm{PCMB}$, and the mutation of Cys184Ser could significantly affect the catalytic activity of the enzyme. This is because these chemical modifications can increase the steric hindrance for the entrance/exit of substrate/products from the active site and because the Cys184Ser substitution can induce the conformational change of the active site due to the higher polarity of the serine, respectively. The location of Cys184 was analyzed using the "hydrophobicity surface" program and described based on the models of the hypothetical substrate entrance/product exit tunnel in the 3D crystal structures of StoEst (11) and PcaEst (10) previously reported. However, the cysteine 
residue, Cys184, in the SsoP1Est is not conserved at the same position in these two esterases (Fig. 1). In addition, the result (Table 1) that the mutation of Cys184Ser inactivated the SsoP1Est was similar to the results that Cys123Ser dienelactone hydrolase (the active site, Cys123) from Pseudomonas knackmussii showed approximately $10-15 \%$ of wild-type activity and a 60 -fold decreased $K_{\mathrm{m}}$ for PNP-acetate (17), that Cys151Ser dienelactone hydrolase (the active site, Cys151) from S. solfataricus P1 showed little activity as compared to wild-type toward the substrate, PNP-caprylate (18), and that each replacement of all three cysteine residues with serine residue in arylesterase from $S$. solfataricus P1 inhibited $70-96 \%$ of wild-type activity (16). The dienelactone hydrolase enzyme belongs to the same $\alpha / \beta$ hydrolase superfamily as serine esterases.

\section{Fluorescence analysis}

To understand the result that each mutation of Cys94Ser, Cys101Ser, Cys103Ser, and Cys184Ser inactivated the enzyme significantly (Table 1), any conformational changes by these mutations were examined by a fluorescence study using two tryptophan residues that existed in the enzyme. As shown in Fig. $2 \mathrm{C}$, each substitution of the four cysteine residues resulted in a red shift of the intrinsic tryptophan fluorescence spectrum. This indicates that at least part of the Trp residues became more exposed to a hydrophilic environment due to the conformational change caused by the Cys substitution. It is not easy, however, to explain how such a conformational change leads to the inactivation of the enzyme unless their 3D structures are determined.

\section{MATERIALS AND METHODS}

\section{Strains, plasmids, and growth conditions}

XL1-Blue supercompetent cells and $E$. coli $\mathrm{DH} 5 \alpha$ ultracompetent cells were used as host cells for mutant plasmids containing the mutated SsoP1Est genes produced by polymerase chain reaction (PCR), whereas E. coli BL21 (DE3) cells harboring all the recombinant plasmids including the mutant plasmids were used for gene expressions. The vector utilized for expression was $\mathrm{pET}-11 \mathrm{~d}$ (Novagen). These $E$. coli $\mathrm{DH} 5 \alpha$ and $E$. coli BL21 (DE3) cells were routinely grown at $37^{\circ} \mathrm{C}$ in Luria-Bertani (LB) medium with ampicillin $(100 \mu \mathrm{g} / \mathrm{ml})$.

\section{Construction of recombinant plasmid DNAs containing mutant SsoP1Est genes}

Standard molecular cloning techniques were employed throughout $(19,20)$. The transformed E. coli BL21 (DE3) cells harboring the recombinant DNA plasmid (pSsoP1Est), which the SsoP1Est gene was incorporated into the BamHI site of the expression vector, pET-11d, were made in a previous study (14). The constructed pSsoP1Est containing the SsoP1Est gene obtained from these transformed cells was used as the template for the mutations (Ser151Ala, Asp244Asn, and His274Asn) of the expected catalytic triad (Ser151, Asp244, and His274), for the mutations (Gly80Glu, Gly81Glu, and Ala152Phe) of the expected oxyanion hole (Gly80, Gly81, and Ala152), and for the mutations (Cys94Ser, Cys101Ser, Cys103Ser, and Cys184Ser) of the expected cysteine residues (Cys94, Cys101, Cys103, and Cys184), which might be related respectively to the catalytic process of the SsoP1Est as described in a previous paper (14), in the SsoP1Est gene by site-directed mutagenesis. As shown in Table S1 in Supplemental data, mutagenic oligonucleotide primers were designed, and PCR based on the QuikChange II site-directed mutagenesis kit (Stratagene Co.) for the construction of SsoP1Est mutant plasmids was carried out using pSsoP1Est as the template DNA and forward- and reverse-primers with lengths of 25 to 42 bases mutated at the desired sites in the SsoP1Est gene, according to the guidelines and protocol recommended by the manufacturer. The PCR conditions were as follows: 1 cycle of 30 s at $95^{\circ} \mathrm{C}$ followed by 18 cycles of $30 \mathrm{~s}$ at $95^{\circ} \mathrm{C}, 1 \mathrm{~min}$ at $55^{\circ} \mathrm{C}$, and $7 \mathrm{~min}$ at $68^{\circ} \mathrm{C}$. The products were treated with $1 \mu \mathrm{l}$ of Dpnl $(10$ $\mathrm{U} / \mu \mathrm{l}$ ) at $37^{\circ} \mathrm{C}$ for $1 \mathrm{~h}$ to digest the template DNA, the PCR products were transformed into XL1-Blue supercompetent cells for nick repair, and then the mutated cells were screened on LB-ampicillin agar plates containing $80 \mu \mathrm{g} / \mathrm{ml}$ 5-bromo-4-chloro-3-indolyl-betaD-galactopyranoside (X-gal) and $20 \mathrm{mM}$ isopropyl-L-D-thiogalactoside (IPTG). The mutant plasmids obtained from these cells were sequenced to confirm the presence of the correct mutations.

\section{Overexpression and purification of mutant enzymes}

The mutant plasmids were transformed into E. coli BL21 (DE3) competent cells for overexpression and cultivated in 1 liter of LB medium supplemented with ampicillin $(100 \mu \mathrm{g} / \mathrm{ml})$ at $37^{\circ} \mathrm{C}$. Transformed cells containing the expression vector plasmid, pET-11d, and the native recombinant plasmid, pSsoEst, were used as controls. When the optical density of cultured cells reached 0.5 at $600 \mathrm{~nm}, 1 \mathrm{mM}$ IPTG was added to induce gene expression. After an additional 5-h cultivation, the overexpressed mutant enzymes from the cultivated transformed cells were purified using twice-heating of cell extracts and Butyl-Sepharose column as described in the purification of the native enzyme in previous study (14). The identification of the mutant enzymes during the purification procedure was performed by a molecular size comparison with the native one on an SDS-PAGE gel.

\section{Enzyme assay and activity staining}

The native and mutant enzyme assay was performed with an artificial chromogenic substrate, $p$-nitrophenyl (PNP) caprylate $\left(C_{8}\right)$, as described in previous study (14). The enzyme reaction at $60^{\circ} \mathrm{C}$ was started by the addition of the purified native recombinant or mutant carboxylesterase enzyme $(12 \mu \mathrm{g} / \mathrm{ml})$. Measurements were carried out at least three times. Standard deviations never exceeded $10 \%$ of the mean values.

Two different activity staining methods carried out on tributyrin-emulsified agar plates and on a gel with $\alpha$-naphthyl acetate $(\alpha-N A)$ after SDS-PAGE were employed for tracing carboxylesterase activity during the purification procedure as described previously (14). 


\section{Determination of molecular mass of native recombinant SsoP1Est and mutant enzymes}

The molecular weight of the purified native recombinant or mutant carboxylesterase was determined on $12.5 \%(\mathrm{w} / \mathrm{v})$ SDS-polyacrylamide gel using low-molecular-weight standards. Gels were stained with silver (21).

\section{Homology modeling of native recombinant SsoP1Est}

To construct a structural model of the native recombinant SsoP1Est, the I-TASSER protocol $(22,23)$ was used. By repeating these procedures several times with the I-TASSER Server, several models were constructed. The structural models with the highest confidence score of these models was selected to compare with that of SsoP1Est. The UCSF Chimera 1.9 program was employed for molecular visualization and analysis.

\section{Fluorescence spectroscopy}

Emission spectra of normal esterase (SsoP1Est) and Ser-mutant esterases (Cys94Ser, Cys101Ser, Cys103Ser, and Cys184Ser) were obtained with a PerkinElmer LS-55 fluorescence spectrometer at a protein concentration of $0.1 \mathrm{mg} / \mathrm{ml}$ in $10 \mathrm{mM}$ sodium phosphate buffer, $\mathrm{pH}$ 7.0. The excitation was set at 298 $\mathrm{nm}$ in order to exclude the tyrosine contribution to the overall fluorescence emission, and the emission was recorded from 300 $\mathrm{nm}$ to $400 \mathrm{~nm}$. The data were transferred to an ASCII file, and the emission spectra were recorded using FL Win Lab software installed in the PerkinElmer fluorescence spectrometer.

\section{ACKNOWLEDGEMENTS}

This study was supported by a 2014 Research Grant from Kangwon National University (No. 120141503), and by the KRIBB Research Institute Program, Republic of Korea.

\section{REFERENCES}

1. Okuda H (1990) Esterases; in A study of enzymes, Kuby SA (eds.), 563-577, Vol. 2, CRC press, Boca Raton, FL, USA

2. Jaeger KE, Dijkstra BW and Reetz MT (1999) Bacterial biocatalysts: molecular biology, threedimensional structures, and biotechnological applications of lipases. Annu Rev Microbiol 53, 315-351

3. Arpigny JL and Jaeger KE (1999) Bacterial lipolytic enzymes: classification and properties. Biochem J 343, 177-183

4. Rao L, Xue Y, Zhou C et al (2011) A thermostable esterase from Thermoanaerobacter tengcongensis opening up a new family of bacterial lipolytic enzymes. Biochim Biophys Acta 1814, 1695-1702

5. Holm C, Osterlund T, Laurell H and Contreras JA (2000) Molecular mechanisms regulating hormone-sensitive lipase and lipolysis. Annu Rev Nutr 20, 365-393

6. Holmquist M (2000) Alpha/beta-hydrolase fold enzymes: structures, functions and mechanisms. Curr Protein Pept Sci 1, 209-235

7. Schrag JD and Cygler M (1997) Lipases and alpha/beta hydrolase fold. Methods Enzymol 284, 85-107

8. De Simone G, Menchise V, Manco G et al (2001) The crystal structure of a hyperthermophilic carboxylesterase from the archaeon Archaeoglobus fulgidus. J Mol Biol 314, 507-518

9. Byun JS, Rhee JK, Kim ND et al (2007) Crystal structure of hyperthermophilic esterase EstE1 and the relationship between its dimerization and thermostability properties. BMC Struct Biol 7:47, 1-11

10. Palm GJ, Fernández-Álvaro E, Bogdanović X et al (2011) The crystal structure of an esterase from the hyperthermophilic microorganism Pyrobaculum calidifontis VA1 explains its enantioselectivity. Appl Microbiol Biotechnol 91, 1061-1072

11. Angkawidjaja C, Koga Y, Takano K and Kanaya S (2012) Structure and stability of a thermostable carboxylesterase from the thermoacidophilic archaeon Sulfolobus tokodaii. FEBS J 279, 3071-3084

12. Panda T and Gowrishankar BS (2005) Production and applications of esterases. Appl Microbiol Biotechnol 67, 160-169

13. Brock TD, Brock KM, Belly RT and Weiss RL (1972) Sulfolobus: a new genus of sulfur oxidizing bacteria living at low $\mathrm{pH}$ and high temperature. Arch Microbiol 84, 54-68

14. Park Y, Choi SY and Lee HB (2006) A carboxylesterase from the thermoacidophilic archaeon Sulfolobus solfataricus P1; purification, characterization, and expression. Biochim Biophys Acta 1760, 820-828

15. Nam JK, Park YJ and Lee HB (2013) Cloning, expression, and characterization of a novel thermostable esterase from the archaeon Sulfolobus solfataricus P1. J Mol Catal B: Enzymatic 94, 95-103

16. Park YJ, Yoon SJ and Lee HB (2008) A novel thermostable arylesterase from the archaeon Sulfolobus solfataricus P1: purification, characterization, and expression. J Bacteriol 190, 8086-8095

17. Pathak D, Ashley G and Ollis D (1991) Thiol protease-like active site found in the enzyme dienelactone hydrolase: localization using biochemical, genetic, and structural tools. Proteins 9, 267-279

18. Park YJ, Yoon SJ and Lee HB (2010) A novel dienelactone hydrolase from the thermoacidophilic archaeon Sulfolobus solfataricus P1: Purification, characterization, and expression. Biochim Biophys Acta 1800, 1164-1172

19. Sambrook J, Fritsch EF and Maniatis T (1989) Molecular Cloning: A Laboratory Manual, 2nd ed., Cold Spring Harbor Laboratory Press, New York, USA

20. Qin Y and Qu Y (2014) Asn124 of Cel5A from Hypocrea jecorina not only provides the $\mathrm{N}$-glycosylation site but is also essential in maintaining enzymatic activity. BMB Rep 47, 256-261

21. Lim CJ, Jeon JE, Jeong SK et al (2015) Growth hormonereleasing peptide-biotin conjugate stimulates myocytes differentiation through insulin-like growth factor-1 and collagen type I. BMB Rep 48, 501-506

22. Roy A, Kucukural A and Zhang Y (2010) I-TASSER: a unified platform for automated protein structure and function prediction. Nat Protoc 5, 725-738

23. Zhang Y (2008) I-TASSER server for protein 3D structure prediction. BMC Bioinformatics 9:40 